\title{
CPD QUESTIONNAIRE
}

\section{Mark each numbered statement as true or false.}

Regarding the semi-automated method for measuring thickness and white matter integrity of the corpus callosum:

1. Diseases affecting cerebral white matter may lead to left-right asymmetries and atrophy of interhemispheric connections, i.e. the corpus callosum.

2. The technique successfully determined values of corpus callosum midline thickness and interhemispheric differences.

3. Future research is not likely to determine normal values for age, but will be able to compare corpus callosum thickness with peripheral white matter volume loss in large groups of patients, using the semiautomated technique.

\section{Regarding sialoblastomas:}

4. Sialoblastomas are rare salivary gland neoplasms that present congenitally or during early infancy, with significant variability in histological range and clinical course.

5. The term 'sialoblastoma' was coined by Taylor to describe these lesions because it conveyed the dysontogenetic character and the site of the tumour.

6. These tumours uncommonly originate in the parotid gland.

7. They may arise in the submandibular or minor salivary gland.

\section{With regard to varicose veins:}

8. Varicose veins is one of the most common diseases in the world.

9. Routine surgical intervention is no longer the principal definitive management for large, unsightly varicose veins.

10. A sub-group of patients develop recurrent varicose veins post surgery or atypical varicose veins.
11. Conventional venography is no longer considered the gold standard of venous imaging.

\section{Rice body formation:}

12. Is a common complication of rheumatoid arthritis.

13. Rice body formation can also occur without any underlying systemic disorder.

14. CT is the modality of choice for discriminating this pathology from synovial chondromatosis.

\section{Osteogenesis imperfecta:}

15. Is a heterogeneous group of genetic bone disorders.

16. Is characterised by decreased bone mass, increased bone fragility and susceptibility to fractures.

17. The severe, perinatal lethal form (Type II) (OMIM 166210) is characterised by bone fragility, with perinatal fractures, severe bowing of long bones, undermineralisation, and death in the perinatal period due to renal failure.

\section{Regarding free intraperitoneal air:}

18. Rigler's sign was first described in 1941 by L G Rigler as a new radiological sign for recognising free air in the peritoneal cavity on supine radiograph.

19. The presence of pneumoperitoneum allows free intra-peritoneal air to be contrasted with intraluminal gas, accentuating the wall of gascontaining viscera.

20. The football sign was first described by Frik du Preez in 1965

We are pleased to announce that effective from this issue, the number of CEUs per test has been increased to 5 .

CPD Instructions:

1. CPD questionnaires must be completed online by going directly (not via Google) to www.cpdjournals.co.za, and registering. You will then receive an email notifying you of your username and password

for subsequent logging on.

2. Read the articles in the journal to find the answers to the questions.

3. After completing the questionnaire, you can check the answers and print your own CPD certificate.

4. Please contact Gertude Fani on 0216817200 or gertrude@hmpg.co.za in the event of queries. 